\title{
INVESTIGATION OF THE RESPONSE OF A MASONRY ARCH RAILWAY BRIDGE USING MEMBRANE EQUILIBRIUM ANALYSIS
}

\author{
CARLO OLIVIERI ${ }^{*}$, SAM H. COCKING ${ }^{2}$, MAURIZIO ANGELILLO ${ }^{1}$ AND MATTHEW \\ J. DEJONG ${ }^{3}$
}
${ }^{1}$ Department of Civil Engineering (DICIV), University of Salerno, Via Giovanni Paolo II, 132, 84084 Fisciano (SA), Italy e-mail: colivieri@unisa.it; mangelillo@unisa.it
2 Department of Engineering, University of Cambridge, Civil Engineering Building, JJ Thomson Avenue 7a, Cambridge, CB3 OFA, UK e-mail: sc740@cam.ac.uk
${ }^{3}$ Department of Civil \& Environmental Engineering, University of California, Berkeley, 777 Davis Hall, Berkeley, CA 94720-1710
e-mail: dejong@berkeley.edu

Keywords: Masonry, Membrane Equilibrium Analysis, Historic Structure, Railway Viaduct

\begin{abstract}
This paper presents an application of Membrane Equilibrium Analysis (MEA) to a historic masonry arch railway bridge in Leeds, United Kingdom. This case study structure is representative of the many masonry arch bridges present on UK and European railway transport networks. It has been chosen because, since 2016, it has been the subject of a detailed Structural Health Monitoring (SHM) campaign, making it an ideal candidate against which to test analytic models. Typically, asset engineers will be responsible for maintaining a large stock of these structures and will lack the time to perform thorough computational analyses. Therefore, simplified approaches, such as MEA, which can offer insight into structural behaviour, have the potential to be highly valuable. This study represents the first step in applying MEA to masonry arch railway bridges.
\end{abstract}

\section{INTRODUCTION}

Masonry arch bridges are important features of railway and road transport networks, throughout the UK and other European countries. However, despite their prevalence, their structural behaviour under working loads is often not understood in detail. As a result, assessments will typically focus on collapse loads, which can be predicted using limit analysis. Serviceability conditions may not be considered directly at all, despite being a driver of structural deterioration.

To address this, asset engineers increasingly prescribe structural health monitoring for their masonry arch structures. One such case is considered here: a masonry railway bridge in Leeds, UK. This bridge has a working life of 150 years and carries a range of local passenger, cross-country, and freight trains. It has experienced historic damage, which was addressed in an intervention in 2015 [1]. Since 2016, two spans, which were the focus of the 2015 remedial works, have been monitored using a range of sensors. These include a network of fibre-optic Fibre Bragg Gratings (FBGs) to measure the distribution of in-plane strains across the arches, in both longitudinal and transverse directions [2].

This sensor network provides detailed measurements of the bridge's response under its working loads, making this an ideal case study for investigating the effectiveness of simplified modelling techniques [3]. One such modelling approach is described here, based on Membrane Equilibrium Analysis (MEA). In this paper, the modelling approach is developed for an arch bridge, based on a 
typical span from the viaduct case study, and observations are made on the model's behaviour when typical working loads are applied. Future work will compare this modelling output in detail against the FBG measurements and will include cracking and deformation analysis. The equilibrium solutions produced by MEA, besides being sufficient to prove that the structure is safe, may be used to assess their possible effect on material deterioration, and can be compared with the crack patterns detected in the structure.

In order to model the equilibrium problem for this special example of a masonry vault structure, the unilateral No-Tension (NT) model is applied in this paper. The unilateral model for masonry appears in the literature around the end of the eighteenth century and in the nineteenth century (see Moseley [4]). In the twentieth century, this model was described rationally by Heyman in [5].

Considering the application of unilateral models to vaults, recent available literature is extensive. Our main inspiration come from the work of the school of Salerno, originating in their paper on the Lumped Stress Method [6], and applied to vaults in the papers by Fraternali et al. [7], Angelillo \& Fortunato [8], Fraternali [9], and recently by Angelillo et al. in [10] and Gesualdo et al. in [11], where the Membrane Equilibrium Analysis is proposed and applied to some simple cases. We also recall the pioneering work by O'Dwyer [12], and work by Block [13], Block et al. [14], Vouga et al. [15], De Goes et al. [16], Block and Lachauer [17], and Miki et al. [18].

MEA can be used as a tool for finding statically admissible stress fields in masonry structures which can be assumed to exhibit membrane behaviour [10]. Heyman's NT assumption for masonry is enforced by ensuring that the stress potential is concave in form. Starting from a potential which satisfies this constraint and depending on a few parameters, a minimum energy solution for the form is then obtained, through optimization of the parameters, for different load conditions that include the dead load and the moving train loads. The forms which are obtained are contained within the thickness
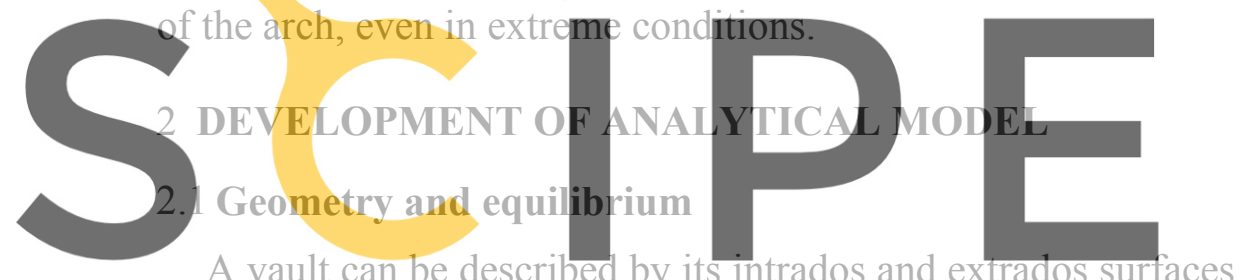

A vault can be described by its intrados and extrados surfaces,
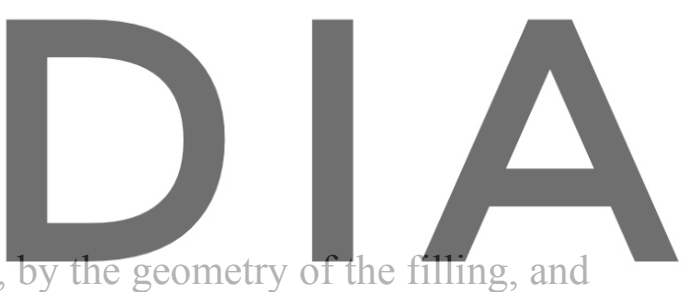

eventually by the form and dimension of the abutments sustaining the vault.

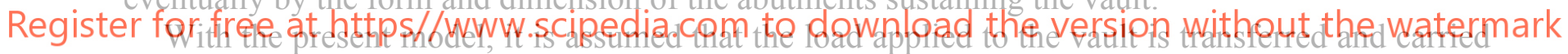

by a membrane structure $S$, a surface to which we may think to attach some thickness $s$. The geometry

of the membrane $S$ is not fixed, in the sense that it can be dispiaced and distorted, provided that it lays inside the masonry; see Fig.1, to which we refer for notation. For the surface $S$ a Monge representation is considered, mapping the position $\mathbf{x}$ of points belonging to $S$ in Cartesian coordinates in the reference shown in Fig.1:

$$
\boldsymbol{x}=\left\{x_{1}, x_{2}, f\left(x_{1}, x_{2}\right)\right\},\left\{x_{1}, x_{2}\right\} \in \Omega,
$$

$\Omega$ being a two-dimensional connected domain, called the planform of $S$, whose boundary $\partial \Omega$, of outer normal $\mathbf{n}$, is composed of a finite number of closed curves; $\left\{x_{1}, x_{2}\right\}$. By adopting the Monge description, the curvilinear coordinates on $S$, are the Cartesian coordinates of points of $S$ in the planform $\Omega ; x_{3}=f\left(x_{1}, x_{2}\right)$ is the rise of the membrane, and we assume: $f \in C^{o}(\Omega)$ - that is to say that the surface $S$, which we consider, is continuous but not necessarily smooth.

An efficient way to describe membrane equilibrium of a thin shell under a load $q$ is the formulation by Pucher [19]. Pucher analysis is based on the introduction of the projected stress components, in terms of which two of the three balance equations can be made independent of the membrane shape. The main notation and the Pucher formulation of the equilibrium problem for a membrane, as used here, have been derived using a Differential Geometry approach. The natural and dual base vectors follow from this, and covariant and contravariant tensor components can also be considered. This Differential Geometry approach is especially helpful when converting the projected stress components into physical generalized stress components on the membrane surface. 

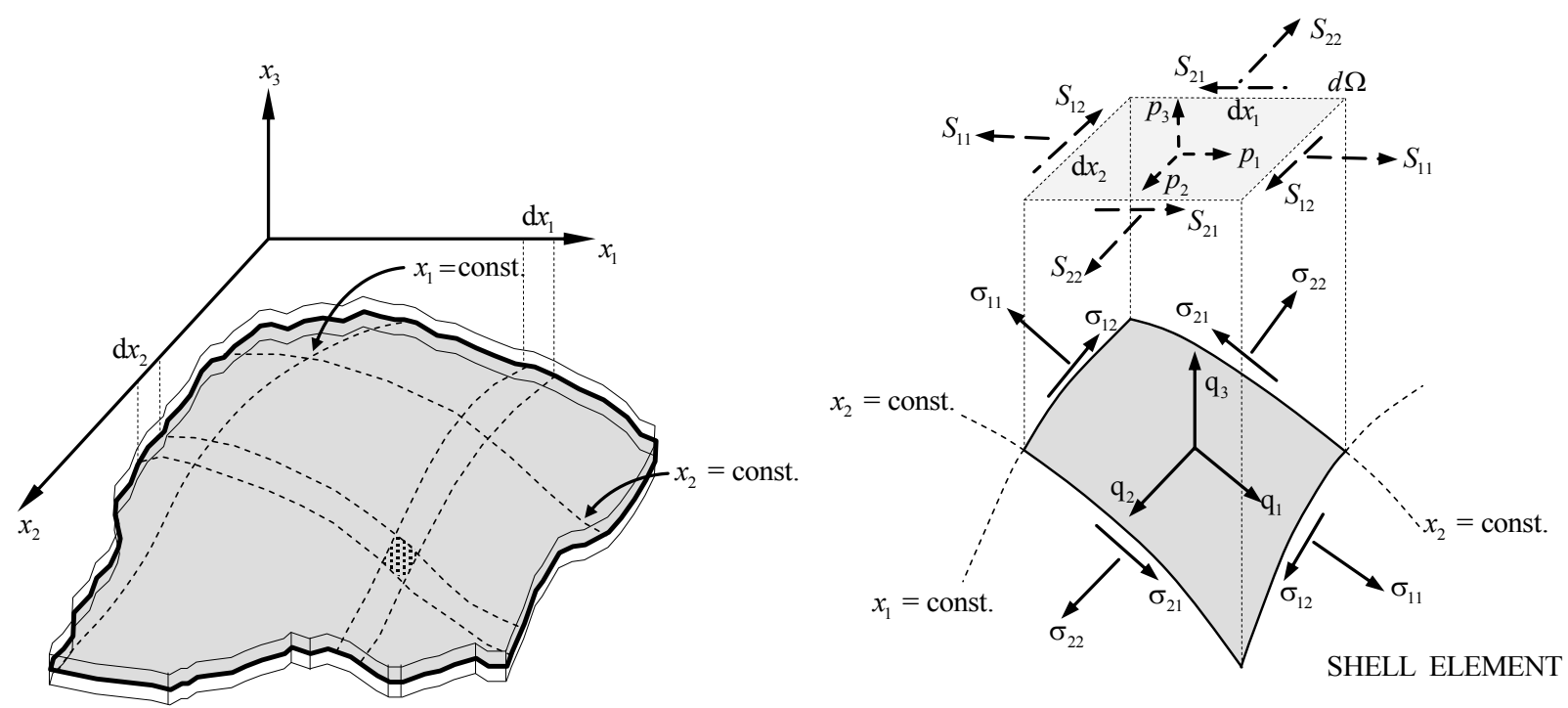

The projected (Pucher) stress components (see Fig.1) are easily defined in terms of the contravariant components $T^{\alpha \beta}$ of the generalized membrane stress: $S_{\alpha \beta}=J T^{\alpha \beta}, J$ being the ratio between the surface area and the projected area. In terms of Pucher stress components, the vector

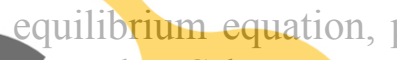
Smat os obceocones

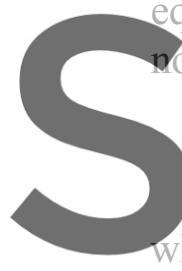

Figure 1: Membrane inside the vault and surface stresses

in the given reference. By using the projected stress, the first two equilibrium equations do not depend

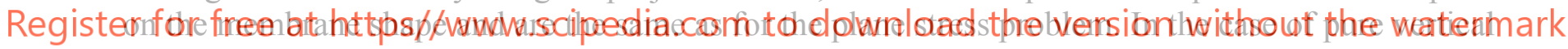
loading, say $p=\{0,0,-p\}$, the first two equations may be solved introducing an Airy stress potential $F\left(x_{1}, x_{2}\right)$ - that here we only assume to be continuous - in the form:

$$
S_{11}=F_{, 22}, S_{22}=F_{, 11}, S_{12}=S_{21}=-F_{, 12} \text {. }
$$

The third equation of transverse equilibrium expresses the balance of the vertical component of the force $p_{3}=-p$ with the scalar product of the Pucher stress matrix times the Hessian of the function $f$. This Hessian matrix is formed by the covariant components of the curvature tensor of the surface. On introducing the Airy stress potential into this equation, we have

$$
F_{, 22} f_{, 11}+F_{, 11} f_{, 22}-2 F_{, 12} f_{, 12}=p \text {. }
$$

\subsection{Unilateral membranes}

A rigid No-Tension (NT) material in the sense of Heyman is assumed, so that the following material restrictions are imposed: the generalized stress $\mathbf{T}$ is negative semi-definite and does no work for the corresponding strain $\mathbf{E}$, that is positive semi-definite:

$$
\boldsymbol{T} \in \mathrm{Sym}^{-} \quad, \quad \boldsymbol{E} \in \mathrm{Sym}^{+}, \boldsymbol{T} \cdot \boldsymbol{E}=0 .
$$

The first application of Pucher's transformation for NT masonry vaults can be found in Angelillo and Fortunato [8], where it is shown that, due to the NT constraint, both the surface generalized stress 
and the matrix of the projected stresses must be negative semi-definite. In terms of the stress function $F$, this condition can be written as:

$$
F_{, 11}+F_{, 22} \leq 0, F_{, 11} F_{, 22}-F_{, 12}^{2} \geq 0
$$

hence, $F\left(x_{1}, x_{2}\right)$ is concave.

\subsection{Singular stress and equilibrium}

If $F$ is only continuous, it may present folds. In this case the projected stress is a line Dirac delta with support along the projection $\Gamma$ of the fold. The Hessian $\mathbf{H}$ of $F$ is singular transversely to $\Gamma$, namely it has a uniaxial singular part parallel to the unit vector $\mathbf{h}$ normal to $\Gamma$. Correspondingly the directional derivative of $F$ in the direction of $\mathbf{h}$, called $F_{h}$, presents a jump. Therefore, the singular part of the Hessian $\mathbf{H}$ of $F$ can be written as:

$$
\boldsymbol{H}_{s}=\delta(\Gamma) \Delta F_{h} \boldsymbol{h} \otimes \boldsymbol{h},
$$

\section{$\delta(\Gamma)$ being the unit line Dirac delta on $\Gamma$ and $\Delta F_{h}$ the jump of slope along the direction $\boldsymbol{h}$. Therefore, the corresponding projected stress (8) has also a singular part, a line Dirac delta on $\Gamma$ :}

$$
S_{S}=\delta(\Gamma) \Delta F_{h} \boldsymbol{k} \otimes \boldsymbol{k},
$$

where $\mathbf{k}$ is the unit vector tangent to $\Gamma$. The concavity of $F$ implies the concavity of the fold whose
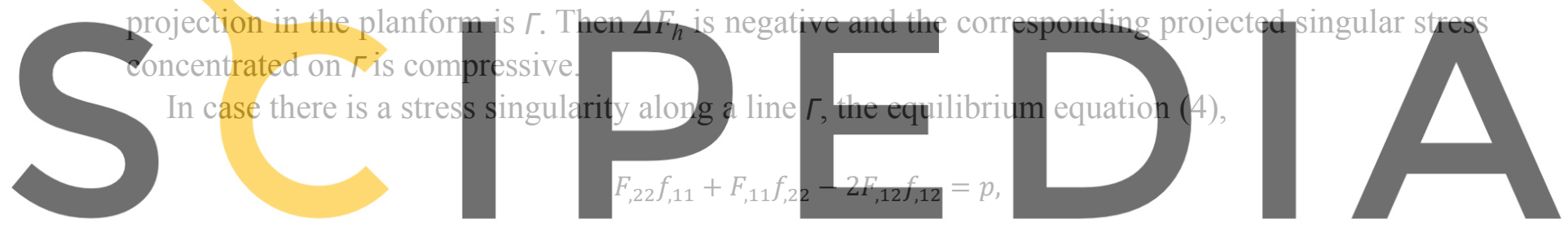

on that line must be reinterpreted in a weak sense. The main conclusion is that if there is a fold in the

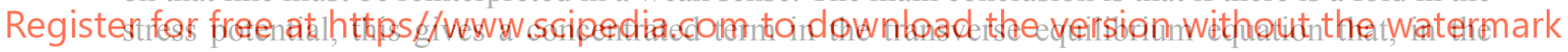

absence of any concentrated load p, must be compensated by another concentrated term. Due to the structure of the equation, this compensation term can only be given by an analogous concentrated term, arising from a fold in the surface whose projection must be the same line $\Gamma$. Since the stress potential is concave, the two concentrated terms can cancel each other only if the Hessian of the surface along the fold is an indefinite matrix (that is the two principal values of it have different signs).

\subsection{Boundary conditions}

Assuming that the shape of the membrane is given, the equilibrium problem for the unilateral membrane $S$, under pure vertical loading, consists in finding a concave stress function $F\left(x_{1}, x_{2}\right)$ satisfying equation (9), with the boundary conditions:

$$
F\left(x_{1}, x_{2}\right)=g\left(x_{1}, x_{2}\right) \quad \text { or } \quad d F / d n=h\left(x_{1}, x_{2}\right) \quad \text { on } \partial \Omega
$$

being $g\left(x_{1}, x_{2}\right)$ and $h\left(x_{1}, x_{2}\right)$ the contact internal moment and axial force produced by the allied tractions, on a beam structure having the same shape as $\partial \Omega$.

Notice that the second order differential equation (4) can be elliptic, parabolic or hyperbolic, depending on the sign of the coefficients, that is whether the shape is locally strictly convex (or concave) or not. If there are regions where the equation is hyperbolic, the characteristics of the equation are real and formulating the problem as a boundary value problem may become impossible.

Since the equilibrium problem is perfectly symmetrical with respect to the two functions 
$F\left(x_{1}, x_{2}\right), f\left(x_{1}, x_{2}\right)$, we may switch the roles of unknowns and data, thinking that the stress, or similarly the stress potential, is given, and that the shape is unknown. The shape can then be found by solving the second order differential equation (4) with the boundary conditions:

$$
f\left(x_{1}, x_{2}\right)=f^{\circ}\left(x_{1}, x_{2}\right), \quad \text { on } \partial \Omega
$$

$f^{\circ}\left(x_{1}, x_{2}\right)$ being the boundary values of the membrane surface. In this case the equation may become at most parabolic, since, due to the No-Tension constraint, the stress potential must be concave.

\section{ANALYSIS OF RAILWAY BRIDGE}

\subsection{Typical span geometry}

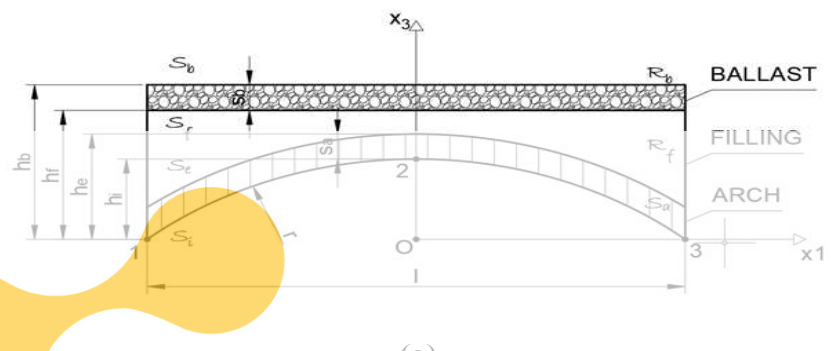

(a)

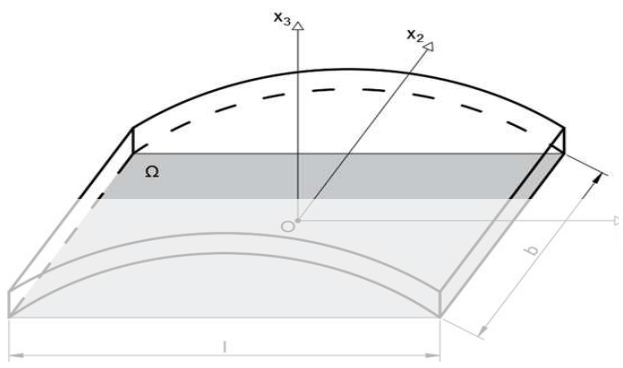

(b)

Figure 2: Schematic section of the bridge (a) and a prospective view of the vault structure (b)

Our case study is a mase years and carries a range historic damage, which w ing a range of sensors, in e distribution of in-plane strain

A schematic section of the bridge is reported in Fig.2a. In Fig.2b a prospective view of the vault

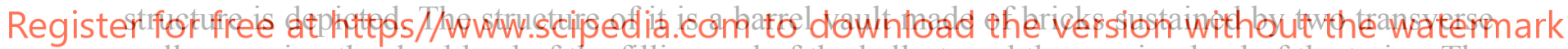
walls, carrying the dead load of the filling and of the ballast, and the moving load of the trains. The filling is confined by two spandrel walls made of bricks. The main geometrical parameters introduced in Fig. 1a have the following values:

$$
\begin{aligned}
b & =8.00 m ; l=7.70 m ; s_{a}=0.50 m ; s_{b}=0.30 m ; \\
h_{f} & =3.05 m ; h_{b}=h_{f}+s_{b} ; h_{i}=1.55 m ; h_{e}=h_{i}+s_{a} .
\end{aligned}
$$

\subsection{Self-weight and train loads}

For the train load we consider, for each train, a total weight $g_{t}=327.5 \mathrm{kN}$, distributed over a small area by considering an exponential distribution (see Fig.3). For the material densities and the gravity acceleration we consider:

$$
\rho_{a}=1500 \mathrm{~kg} / \mathrm{m}^{3}, \rho_{f}=1800 \mathrm{~kg} / \mathrm{m}^{3}, \rho_{b}=1600 \mathrm{~kg} / \mathrm{m}^{3}, \mathrm{~g}=9.81 \mathrm{~m} / \mathrm{s}^{2} .
$$

\subsection{Solution (two trains passing on the bridge span)}

In this study, we assign a form to the stress potential, which depends on a few parameters, and look for the equilibrium solution for the shape by parametrically solving equation (4). If we consider an elementary arch-like solution, recalling that the projected stress is uniaxial and constant in the direction $x_{1}$, the surface which solves the differential equation is a catenary of the load depending on a single parameter: the thrust per unit length. Due to the effect of the intensely localized loads, i.e. the trains, such a surface is not easily fitted inside the intrados and extrados surfaces of the vault and 
a four cylindrical-hinge mechanism may be created. The idea here is to consider a biaxial stress regime for the projected stresses which is constant and directed as the Cartesian axes $x_{1}, x_{2}$ and depends on two parameters: the intensity of the normal stress in the $x_{1}$ direction and the ratio between the normal stresses in the $x_{2}, x_{1}$ directions.

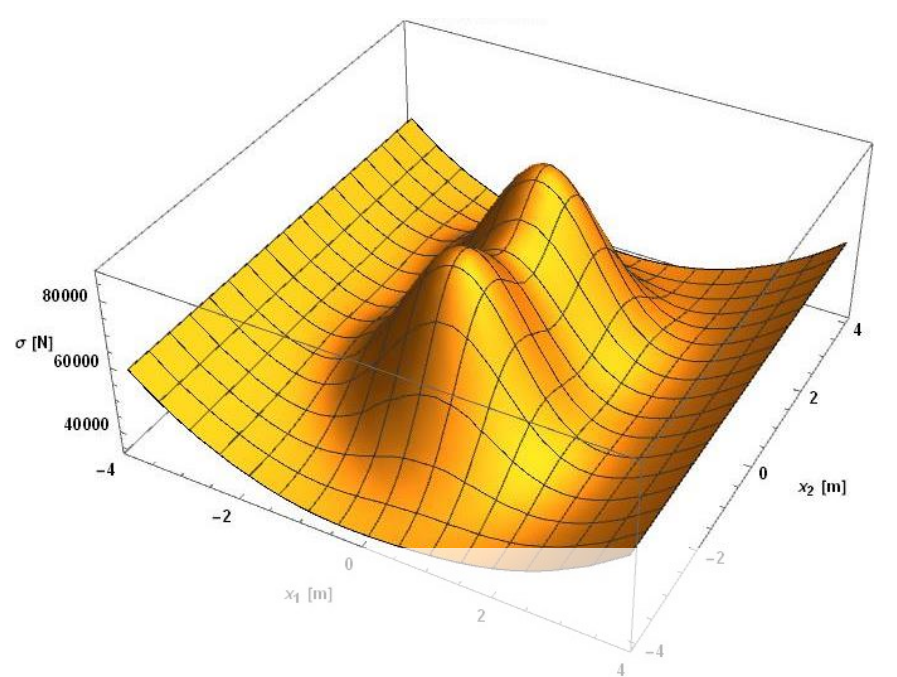

Fig.3: Load profile representing the effect on the membrane of the dead load and the moving load. The moving load due

Since the two sides of the barrel vault parallel to the $x_{1}$ direction are traction free, the $x_{2}$ component of the projected normal

component and bring it to

forming an arch in the plan
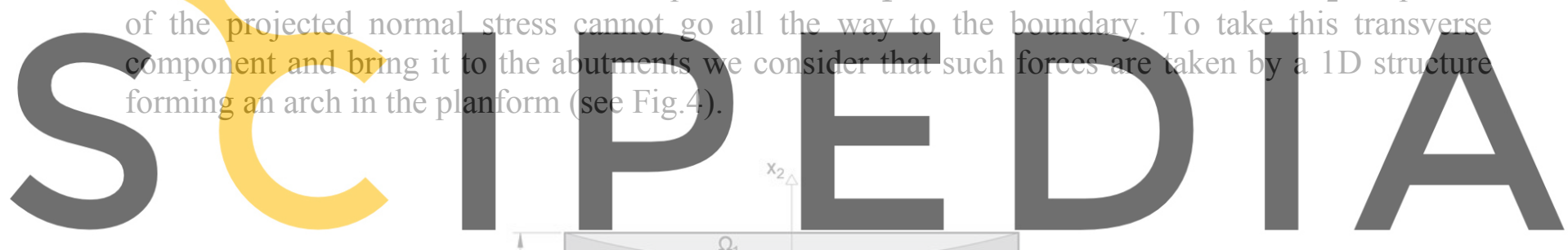

Register for free at https//www.scipedia.com to download the version without the watermark

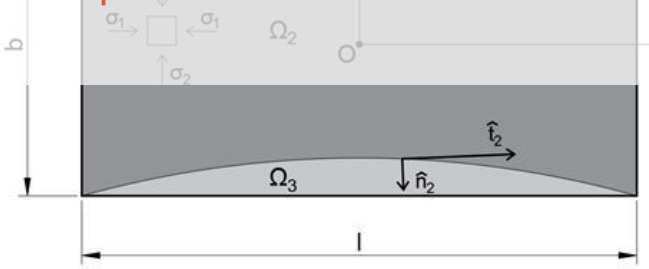

Fig.4: Projected stresses in the planform. The stress is constant and biaxial in the directions of the axes. The jump of the stress across the two curved lines is balanced by a concentrated axial stress acting along them.

To construct this arch and, at the same time, deriving the concentrated axial force along the arch itself, we cut the Airy stress function associated with these stresses, using two symmetric slanted planes, as shown in Fig.5. These planes, parallel to the $x_{1}$ direction, are defined by their height $\mathrm{H}$ at the origin. 


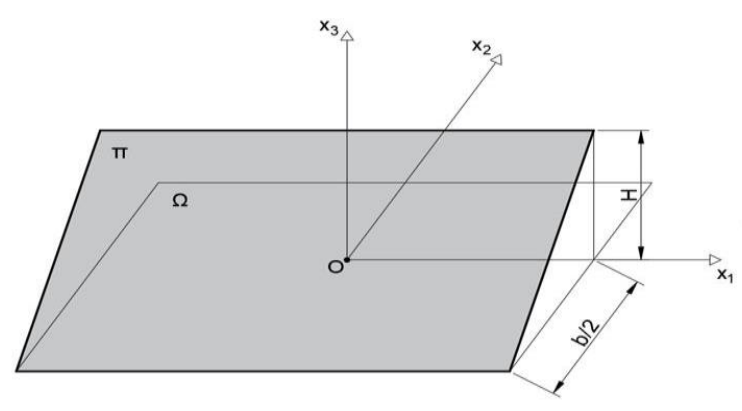

(a)

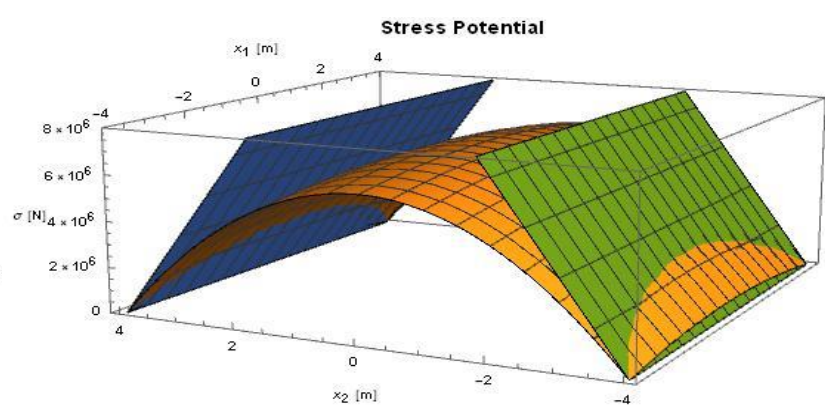

(b)

Fig.5: One of the two symmetric planes intersecting the stress function (a) and intersection of the Airy stress function with the two planes (b).

The Airy stress function associated with the biaxial stress regime depicted in Fig. 6 is defined as follows

$$
F=\frac{\sigma}{8}\left(\left[b^{2}-4 x_{2}^{2}\right]+\alpha\left[l^{2}-4 x_{1}^{2}\right]\right)
$$

$\sigma$ and $\alpha$ are two parameters, the first controlling the intensity of the stress and the second controlling the ratio between the two normal stress components. By intersecting this stress potential with the two planes, we obtain two plane curves whose projections on the planform give the two 1D arch structures on which the axial force is attached. These two arches also define the domain $\Omega$ (see Fig.6) on which
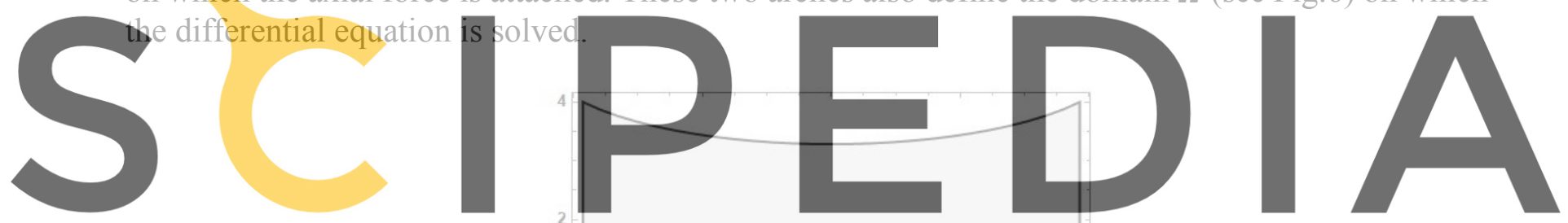

Register for free at https//www.scipedia.com to download the version without the watermark

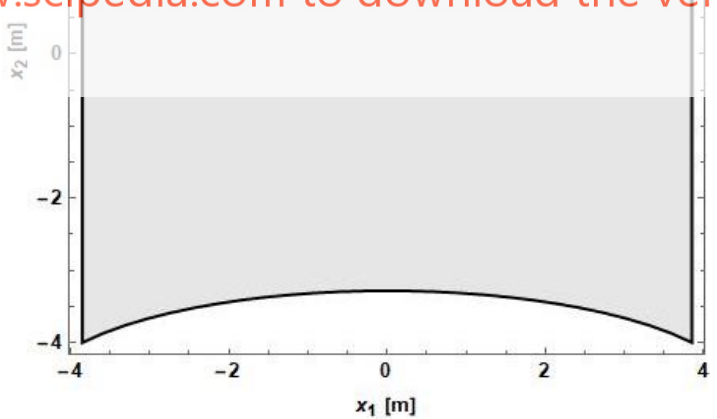

Fig.6: Domain where the transverse equilibrium equation is solved numerically.

We numerically solve the second order (elliptic) differential equation (4) by employing tools in the Wolfram Program Mathematica (see [20]), for finite element solution. We define $f_{m}$, a convenient reference surface contained inside the vault, and choose it to be $10 \%$ of the thickness $(5 \mathrm{~cm})$ above the intrados surface (see Fig.7). In this way, we can consider the thickness of the thrust surface to be $10 \mathrm{~cm}$. 


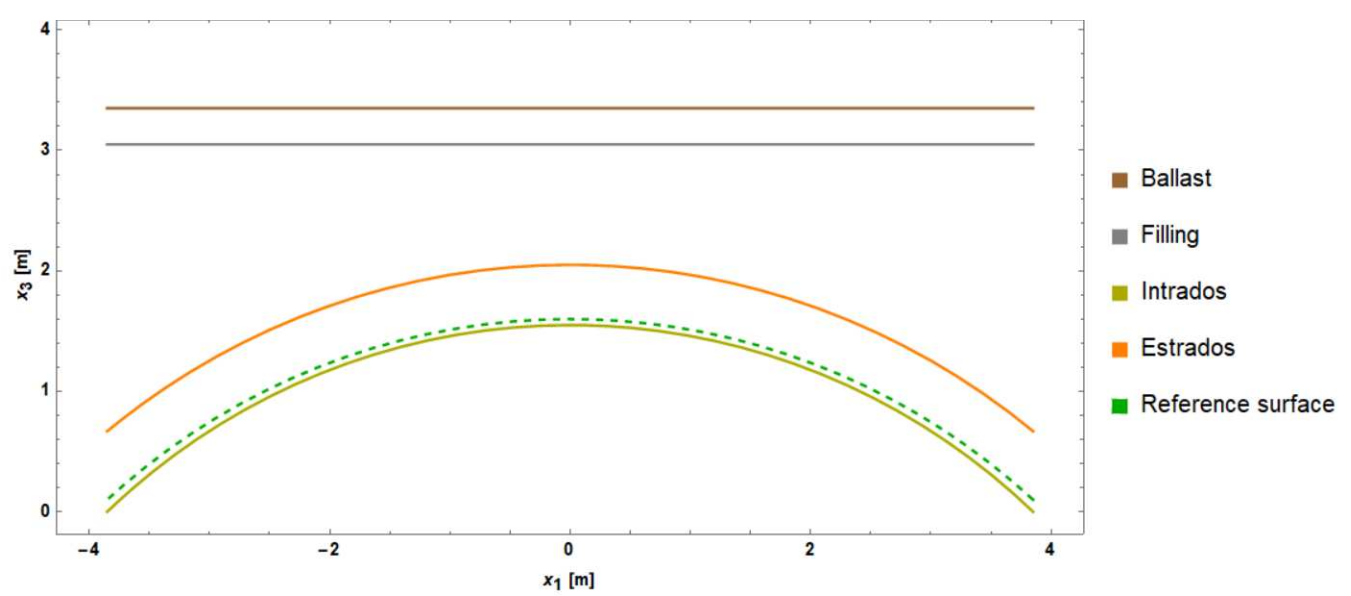

Fig.7: Bridge geometry, axial section.

By using the Finite Element tool in Mathematica, the equation is solved parametrically for the shape $f$ with respect to the parameters $\sigma, \alpha, H$, by considering the Dirichelet data for $f$ :

$$
f\left(x_{1}, x_{2}\right)=f_{m}\left(x_{1}, x_{2}\right), \quad \forall\left(x_{1}, x_{2}\right) \in \partial \Omega
$$

\section{Finally, the parameters $\sigma, \alpha, H$ are optimized by minimizing the objective function}

$$
I=1 / \operatorname{ar}(\Omega) \int_{\Omega}\left(f(\sigma, \alpha, H)-f_{m}\right)^{2} d x_{1} d x_{2}
$$

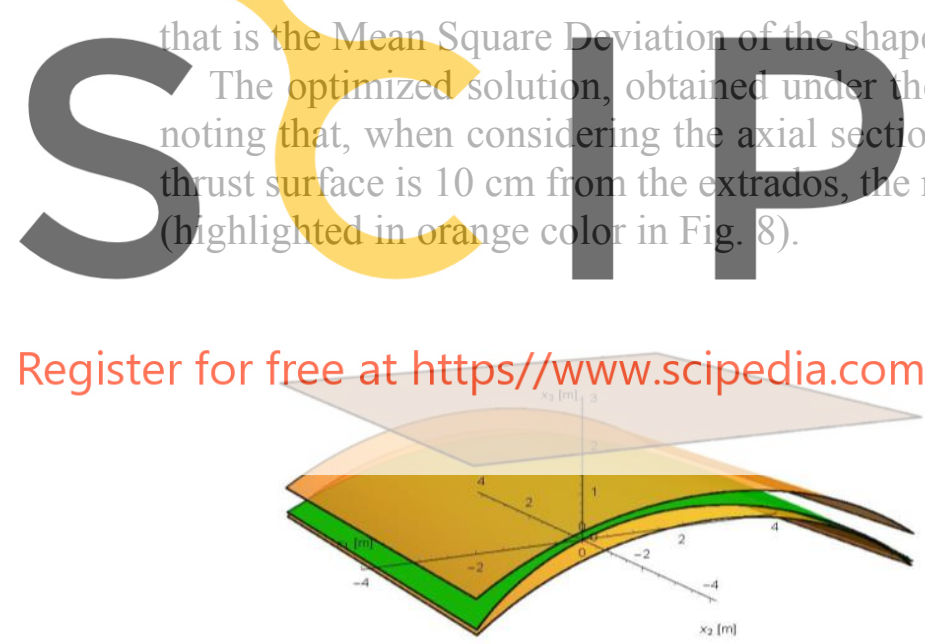

(a)

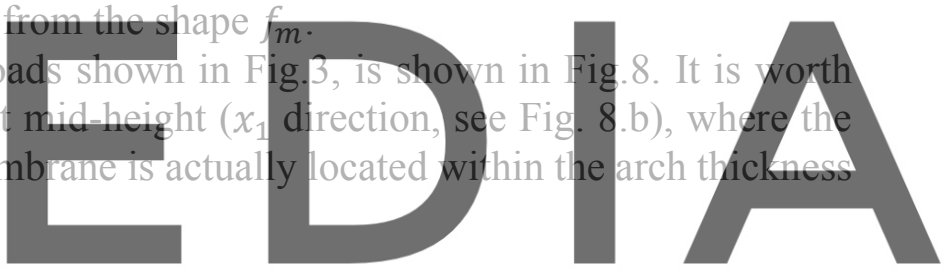

\section{to download the version without the watermark}

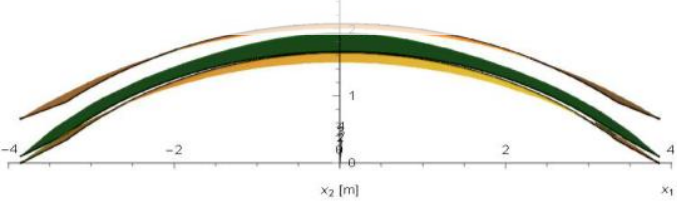

(b)

Fig.8: Thrust surface obtained through optimization inside the bridge geometry: perspective view (a) and axial section at mid-height in $x_{1}$ direction (b).

This solution gives two constant projected stresses in the $x_{1}, x_{2}$ directions: $\sigma_{1}=763 \mathrm{kN} / \mathrm{m}$ and $\sigma_{2}=82 \mathrm{kN} / \mathrm{m}$. Considering a thickness of $10 \mathrm{~cm}$, the acting stress inside the arch is $7.6 \mathrm{MPa}$, which is below the allowable compressive strength of the bricks. The thrust at the four corners of the two arches, $R=649 \mathrm{kN}$, is inclined at the tangent to the arches at those points. The values of these thrust components in the directions $x_{1}, x_{2}$ are, respectively, $R_{1}=568 \mathrm{kN}$ and $R_{2}=314 \mathrm{kN}$. Note that this is one feasible solution, but further optimization could reduce these forces.

\subsection{Solution (one train passing on the bridge span)}

Now we consider, in addition to the dead load, the weight of a single train on one side of the bridge (see Fig. 9). 


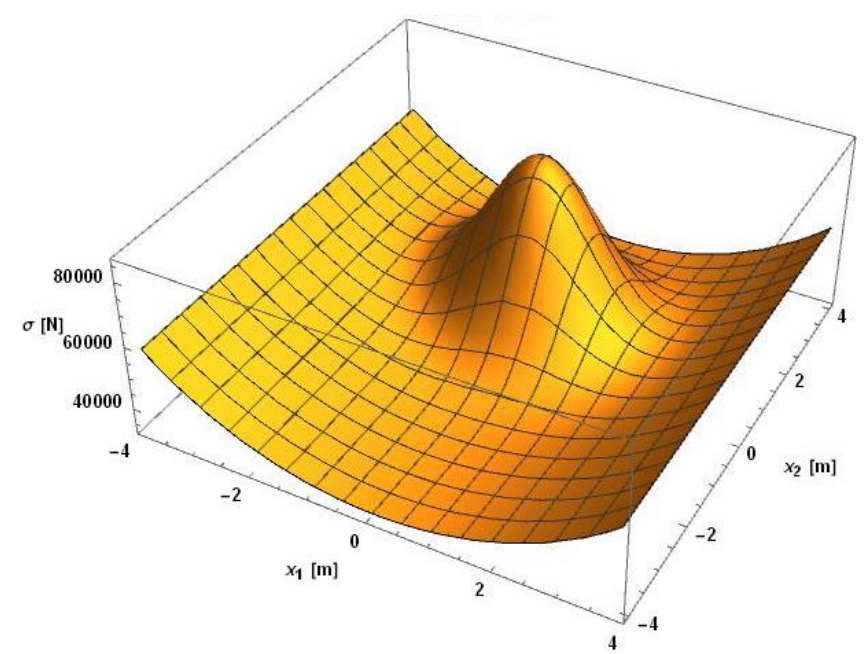

Fig.9: Load profile representing the effect on the membrane of the dead load and the moving load. The moving load due to the train is considered here applied at mid span on one railway.

\section{The optimized solution under the load shown in Fig.9, is shown in Fig.10.}
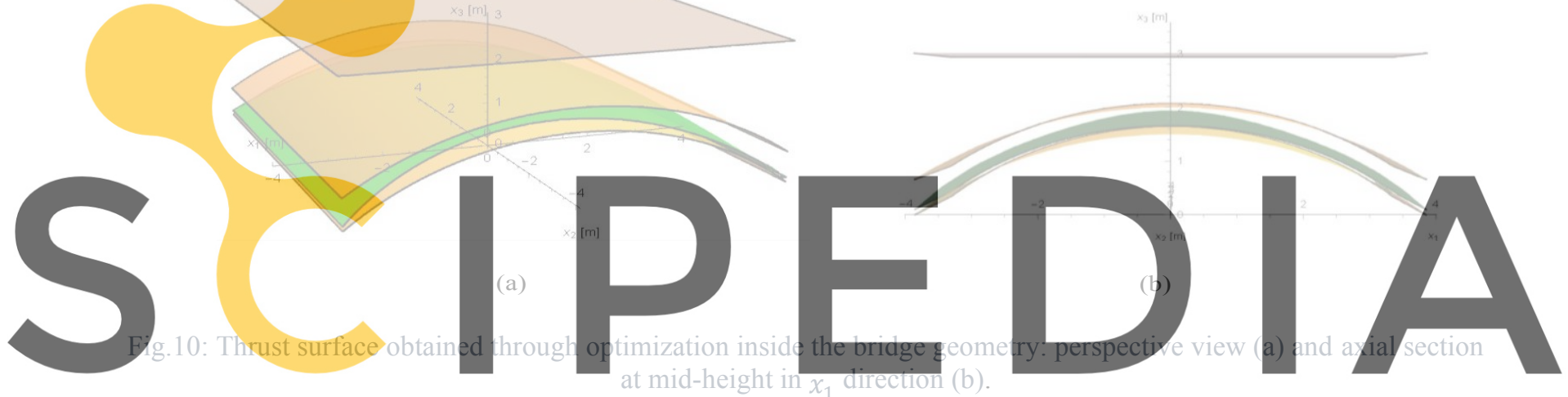

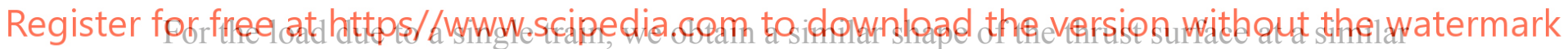

distance from the extrados. However, now the solution gives two constant projected stresses in the $x_{1}, x_{2}$ directions: $\sigma_{1}=598 \mathrm{kN} / \mathrm{m}$ and $\sigma_{2}=64 \mathrm{kN} / \mathrm{m}$. Considering the same thickness of $10 \mathrm{~cm}$, the acting stress inside the arch is now $6.0 \mathrm{MPa}$, and the thrust at the four corners of the two arches, $R=509 \mathrm{kN}$, is inclined at the tangent to the arches at those points. The values of these thrust components in the directions $x_{1}, x_{2}$ are, respectively, $R_{1}=446 \mathrm{kN}$ and $R_{2}=246 \mathrm{kN}$.

\section{FINAL REMARKS}

Analyzing these two solutions, which characterize typical working conditions for this bridge, we observe that, for the same load profile, we can obtain a class of solutions by changing the distance of the reference surface $f_{m}$ and, consequently, the thickness of the membrane. By increasing this distance, we will certainly find greater projected tensions $\sigma_{1}$ and $\sigma_{2}$ but the consequent increase in thickness will give us lower stresses inside the arch.

Future development might include investigation of the optimum $f_{m}$, that gives the true proportion between the position of $f_{m}$, the consequent thickness of the membrane, and the stress inside the arch.

Acknowledgements. The authors wish to acknowledge the work of Isabella Elia, who assisted in constructing the analytical model.

\section{References}

[1] Alexakis, H., Franza, A., Acikgoz, S. and DeJong, M.J. Monitoring bridge degradation using 
dynamic strain, acoustic emission and environmental data. In: Proceedings of the International Conference on Smart Infrastructure and Construction (ICSIC), Cambridge, UK (2019), pp. 523-532. https://doi.org/10.1680/icsic.64669.523

[2] Acikgoz, S., DeJong, M.J., Kechavarzi, K., and Soga, K. Dynamic response of a damaged masonry rail viaduct: Measurement and interpretation. Engineering Structures (2018) 168: 544-558. https://doi.org/10.1016/j.engstruct.2018.04.054

[3] Cocking, S., Acikgoz, S. And DeJong, M.J. Interpretation of the Dynamic Response of a Masonry Arch Rail Viaduct Using Finite-Element Modeling. J. Archit. Eng. (2020)

26(1):05019008. https://doi.org/10.1061/(ASCE)AE.1943-5568.0000369

[4] Moseley, H. On a new principle in statics, called the principle of least pressure. London and Edinburgh Philosophical Magazine and Journal of Science (1833), 3:285-288.

[5] Heyman, J. The stone skeleton. Int J Solids Struct (1966), 2(2):249-279.

[6] Angelillo, M., Fortunato, A., Fraternali, F. The Lumped Stress Method and the DiscreteContinuum Approximation. Int J Solids Struct (2002), 39:6211-6240.

[7] Fraternali, F., Angelillo, M., Rocchetta, G. On the Stress Skeleton of Masonry Vaults and Domes. In: Proceedings of the Seventh Pan American Congress of Applied Mechanics (PACAM VII) Temuco, Chile (2002), pp. 369-372.

[8] Angelillo, A., Fortunato, A. Equilibrium of masonry vaults. In: Lecture notes in applied and computational mechanics, Novel approaches in civil engineering (Eds Fremond, M., Maceri, F.) (2004), 14:105-111. Springer, Berlin.
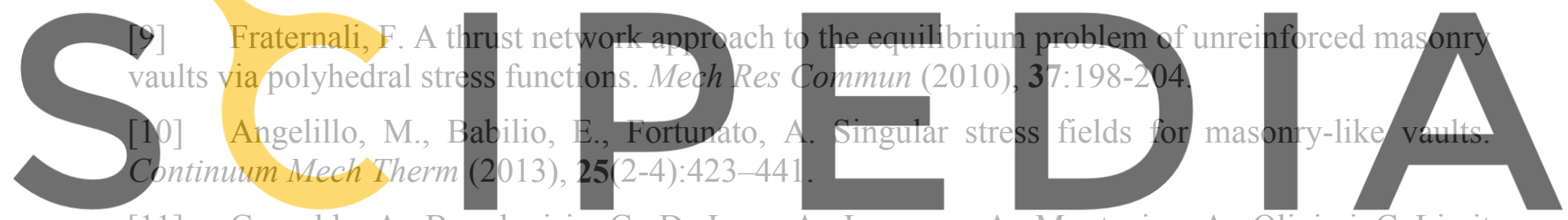

Gesualdo, A., Brandonisio, G., De Luca, A., Iannuzzo, A., Montanino, A., Olivieri, C. Limit

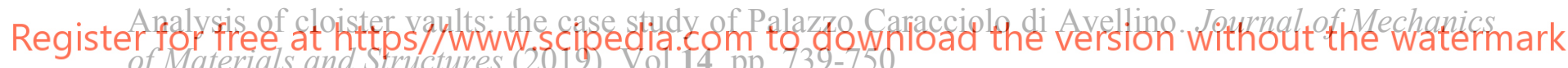

[12] O'Dwyer, D.W. Funicular analysis of masonry vaults. Comput Struct (1999) 73:187-197.

[13] Block, P. Thrust Network Analysis: Exploring Three-dimensional Equilibrium (PhD Dissertation). Massachusetts Institute of Technology, Cambridge, USA (2009).

[14] Block, P., Ciblac, T., Ochsendorf, J. Real-time limit analysis of vaulted masonry buildings. Comput Struct (2006), 84(29-30):1841-1852.

[15] Vouga, E., Hobinger, M., Wallner, J., Pottmann, H. Design of Self-supporting Surfaces. $A C M$ Trans Graph (2012), 31(4): article 87 (11 pages).

[16] De Goes, F., Alliez, P., Owhadi, H., Desbrun, M. On the Equilibrium of Simplicial Masonry Structures. ACM Trans Graph (2013) 32(4): article 93 (10 pages)

[17] Block, P., Lachauer, L. Three-dimensional funicular analysis of masonry vaults. Mech Res Commun (2014), 56: 53-60.

[18] Miki, M., Igarashi, T., Block, P. Parametric Self-supporting Surfaces via Direct Computation of Airy Stress Functions. ACM Trans Graph (2015), 34(4): article 89 (12 pages).

[19] Pucher, A. Über der spannungzustand in gekrümmten flächen. Beton u Eisen (1934), 33:298304.

[20] Wolfram, S., The Mathematica Book, 5th ed., Wolfram Media (2003). 